\title{
Is inhaled budesonide an effective treatment for patients with mild early symptoms of COVID-19?
}

\author{
Hashim Kareemi $^{1}$ (D) Hans Rosenberg ${ }^{1} \cdot$ Krishan Yadav $^{1,2}$ \\ Received: 29 April 2021 / Accepted: 15 May 2021 / Published online: 19 July 2021 \\ (C) The Author(s), under exclusive licence to Canadian Association of Emergency Physicians (CAEP)/ Association Canadienne de Médecine d'Urgence \\ (ACMU) 2021
}

Keywords COVID- $19 \cdot$ Budesonide $\cdot$ Steroids $\cdot$ RCT

\begin{abstract}
Full Citation: Ramakrishnan S, et al. Inhaled budesonide in the treatment of early COVID-19 (STOIC): a phase 2, open-label, randomised controlled trial. Lancet Respir Med. 2021 Apr 9:S2213-2600(21)00160-0. Epub ahead of print.
\end{abstract}

Abstract Link: https://doi.org/10.1016/S2213-2600(21) 00160-0

Article Type: Therapy

Ratings: Methods-2.5/5, Usefulness-2/5

\section{Introduction}

\section{Background}

Early reports of COVID-19 patients showed underrepresentation of those with asthma and chronic obstructive pulmonary disease. The authors hypothesized this was due to a potential protective effect of inhaled glucocorticoids.

\section{Objective}

To compare the effect of an inhaled glucocorticoid (budesonide) versus usual care on adults with early COVID-19 disease.

Hashim Kareemi

hkareemi@toh.ca

1 Department of Emergency Medicine, University of Ottawa, Ottawa, ON, Canada

2 Ottawa Hospital Research Institute, Ottawa, ON, Canada

\section{Structured methods}

\section{Design}

Randomized, open-label, parallel-group clinical trial.

\section{Setting}

Community (Oxfordshire, UK).

\section{Subjects}

Adults ( $>18$ years) with symptoms of COVID-19 (new cough and fever or anosmia, or both) within 7 days of onset. Excluded if recent ( $<7$ days) use of inhaled or systemic glucocorticoids, or known allergy or contraindication to inhaled budesonide.

\section{Intervention}

Budesonide powder inhaler $800 \mu \mathrm{g}$ twice daily.

\section{Control}

Supportive care (antipyretics for fever and honey for cough).

\section{Outcomes}

Primary: composite of COVID-19 related presentation to urgent care, emergency department (ED), or hospitalization. Secondary: self-reported time to symptom resolution, viral symptoms, blood oxygen saturation, body temperature, SARS-CoV-2 viral load. 


\section{Main results}

Among 146 participants in the intention to treat analysis, the primary outcome occurred in $11 / 73(15 \%)$ participants in the usual care group and $2 / 73(3 \%)$ in the budesonide group (difference in proportion $0.123,95 \%$ CI $0.033-0.213$; $p=0.009)$. The trial was stopped early due to reduced recruitment after a national lockdown and determining the study outcome would not change with further enrollment. For secondary outcomes, the budesonide group had shorter self-reported time to symptom resolution (median 7 days [95\% CI 6-9\%] versus 8 days [7-11]; log-rank test $p=0.007$ ) and less days of documented fever $\left(\geq 37.5{ }^{\circ} \mathrm{C}\right)$ during the first 14 days (mean $2 \%$ [SD 6] versus $8 \%$ [18]). There was no significant difference between budesonide and usual care groups in any other secondary outcomes, including proportion of days with oxygen saturation (using home monitor) of $94 \%$ or less during the first 14 days (19\% [SD 24] versus $22 \%$ [27]; $p=0.627$ ).

\section{Appraisal}

\section{Strengths}

- Important clinical question

- Randomized controlled trial

- Inexpensive, widely available therapy

- Speed of recruitment and implementation early in COVID-19 pandemic

\section{Limitations}

- Composite outcome: the individual outcomes are not equivalent. The need for hospitalization indicates a more severe illness and is more clinically relevant than an urgent care or ED visit. Furthermore, the proportion of patients with each of these specific outcomes was not reported.

- Open label: patients knowing they are receiving study drug introduces risk of reporting bias (i.e. patients assigned to the treatment arm may have overly optimistic reports of the effects of the treatment). This is particularly important when many of the outcomes are subjective.

- Low number of primary outcome: 11 patients, of which only 9 had confirmed COVID-19 infection.
- Small sample size: despite meeting a priori stopping rules, the trial being stopped early resulted in a small sample size that limits generalizability.

- Non-blinded analysis

\section{Context}

In the ongoing PRINCIPLE trial assessing inhaled budesonide versus usual care for patients in the community at risk for poor outcomes (i.e. age $\geq 65$ or age $\geq 50$ plus comorbidities) with suspected COVID-19 [1], the most recent interim analysis (March 25, 2021) showed a shorter time to selfreported recovery in the budesonide group (hazard ratio 1.208 [95\% BCI $1.076-1.356$ ], probability of superiority 0.999 , estimated benefit [95\% BCI] of 3.011 [1.134-5.41] days), but not in the rate of hospitalization or death $(59 / 692$ (8.5\%) budesonide versus 100/968 (10.3\%) usual care; estimated percentage benefit, $2.1 \%$ [95\% BCI $-0.7 \%-4.8 \%$ ], probability of superiority 0.928 ).

\section{Bottom Line}

The question posed by the authors is critically important. However, the authors do not report how many patients experienced the most important patient outcome: subsequent hospitalization due to clinical deterioration. The open-label design leaves the patient reported outcomes subject to possible bias. Stopping the trial early, despite best efforts by the authors, contributes to our reticence to support the intervention. Based on these limitations, this study — while showing a signal - does not provide enough evidence to warrant routine use of inhaled glucocorticoids for early COVID-19 disease.

\section{Declarations}

Conflict of interest The authors have no conflicts of interest to declare.

\section{References}

1. YuBafadhel LM, Dorward J. Inhaled budesonide for COVID-19 in people at higher risk of adverse outcomes in the community: interim analyses from the PRINCIPLE trial. MedRxiv. 2021. https://doi.org/10.1101/2021.04.10.21254672. 